\title{
Increasing the accessibility of PhET Simulations for students with disabilities: Progress, challenges, and potential
}

\author{
Katherine K. Perkins and Emily B. Moore \\ Department of Physics, University of Colorado Boulder, UCB 390, Boulder, CO, 80309
}

\begin{abstract}
Despite the potential of PhET Interactive Simulations to foster engagement and participation in science education, they are currently inaccessible for many students with disabilities. This is due to the reliance on predominantly visual representations of concepts and interfaces that rely on dexterity with a mouse or touchscreen device. In 2014, the PhET project began an initiative to increase the accessibility of PhET's HTML5 simulations for students with and without disabilities, using inclusive design approaches. Since then, we have overcome technical challenges, started a (growing) accessible simulation design community, published the first keyboard and screen reader accessible PhET simulation, and engaged in research of inclusive features with students with diverse needs. Here, we share our progress, challenges, and the potential of creating accessible - and inclusive - educational resources for physics classrooms.
\end{abstract}

\section{INTRODUCTION}

The American Physical Society adopted a statement in 2013 regarding K-12 education calling for policy makers, schools, and educators to "provide every student access to high-quality science education.” Unfortunately, many students with disabilities are not receiving access to highquality science education $-8^{\text {th }}$ grade students with disabilities are more than twice as likely to be below basic achievement in science than their peers without disabilities [1]. To provide high-quality access to science education for all students, science education learning materials need to meet the needs of students with and without disabilities. In this paper, we share work in progress - describing efforts by the PhET Interactive Simulations project to build the community, practices, and infrastructure to create inclusive interactive resources, including simulations. We also introduce challenges to the advancement of education research inclusive of students with disabilities, and invite the broader physics education research community to join efforts to develop and investigate learning opportunities for this often overlooked student population.

The vast majority of US K-12 students with disabilities are taught physical science or physics in general education classrooms, rather than in specialized settings. Science teachers are often overwhelmed with the challenge of preparing appropriately differentiated instruction for the diversity of students in their classroom [2]. Inclusive digital learning materials that can be adapted on-the-fly to meet the needs of each student in the classroom would significantly decrease the difficulty, cost, and time required to prepare differentiated instruction for students with disabilities, while increasing opportunities for all students to engage in collaborative science practices. Inclusive learning materials can also provide additional benefits by supplying features that can be beneficial for all students, regardless of ability.
Additionally, increases in investigations and inquiries by the US Dept. of Justice regarding a lack of accessibility of digital educational resources [3] has resulted in increased urgency to understand how to effectively and efficiently implement accessibility features into the increasingly complex digital resources used in today's classrooms.

To help address the challenges of creating accessible interactive physics resources, the PhET Interactive Simulations project [4] has engaged in an initiative to design, develop, and research inclusive physics simulations (sims). By engaging in foundational research, creating exemplars, sharing guidelines, representing interactive resources within the accessibility field, and developing a network of content developers creating inclusive resources, the PhET project is leading efforts to create inclusive features for highly interactive digital learning resources. We envision a future where all interactive physics learning resources are accessible, and capable of supporting students with disabilities in inclusive physics classrooms. Importantly, we envision a community effort to create the research foundations, design practices, and technical infrastructure to support physics instructors and curriculum developers in making their learning materials more inclusive.

\section{ACCESSIBLE PHET SIMULATIONS}

In May of 2017, the popular PhET sim John Travoltage [5] - featuring the character John, who can be shocked by rubbing his foot on a rug and moving his arm toward a doorknob - was given a significant update. With this update, the sim received two newly developed accessibility features, alternative input and auditory descriptions (see Fig. 1) and became the first PhET sim accessible to students with visual impairments or mobility impairments. These features provide keyboard (and other assistive device) input capabilities for students with mobility impairments who 
cannot use a mouse, track pad, or touch screen, and provide non-visual access for students who are blind or visually impaired and utilize screen reader software to read aloud descriptions of onscreen content. These accessibility features result in a more inclusive John Travoltage sim by supporting a broader diversity of student needs through accessibility layers that can be accessed when needed.

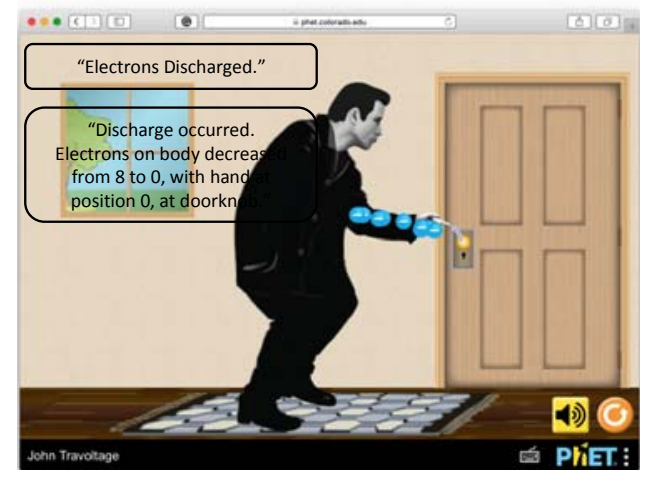

FIG 1. Students can use screen reader software to interact with and hear descriptions of the John Travoltage sim. Example descriptions are shown in text boxes on sim image.

\section{PROGRESS}

The publication of a more accessible John Travoltage, and future accessible sims, required significant advances in understanding how students with disabilities utilize sims, and new design approaches and technical infrastructure to enable sim interaction through assistive technology.

Research. Since 2014, we have conducted research to understand how students with disabilities interact with digital resources. We regularly conduct interviews with blind learners to understand how to create engaging and informative text descriptions that can be read aloud by screen reader software to support exploration, experimentation, and scientific reasoning - providing non-visual, but still fun, effective, and efficient, access to PhET sims [6-8]. For example, we have investigated the relationship between our visual design approach, implicit scaffolding, and the development of descriptions for blind or visually impaired learners [7]. We found that for the blind learners we worked with, a consistent pattern of sim use emerged - starting with intense scanning (quick navigation and listening through the available text descriptions), followed by iterative cycles of interaction with objects in the sim and targeted scanning of specific descriptions. As they used the sim (when no significant technical barriers were encountered), the learners engaged in exploration, asking conceptual questions, and experimentation to find their own answers - similar to the sim use of the many sighted students we have interviewed.

We also conduct interviews with students with low vision and students with intellectual disabilities to learn about the unique accessible technology needs of these students, as well as to locate overlaps in features that provide benefits across different student populations. For example, text-to-speech capabilities, where onscreen labels can be clicked, tapped, or hovered over to be read aloud on demand, could be beneficial for some learners with low vision and for learners with intellectual disabilities who have difficulty reading.

Findings from these research investigations are used to understand navigation approaches used by students with different needs, to identify accessibility challenges unique to interactive learning environments, and to develop guidelines for creating effective accessibility features for sims.

Design. As part of an iterative design process involving our research partners at OCAD University and Georgia Tech, and community members with disabilities, our work aligns with prior research in description for interactive scientific graphics [9], utilizes existing international accessibility web standards [10], and has resulted in new approaches to designing alternative access to highly visual sims $[6,8]$.

The screen reader accessible sims provide a Scene Summary (a brief description of the sim screen), as well as an always up-to-date list of all available sim objects and features and their current state. This information is accessible at any time during a student's interaction using standard keyboard shortcuts for screen reader software. As the student interacts with objects in the sim, they hear real-time alerts that provide concise and pedagogically relevant information about changes to objects, relationships, and readouts.

Through interviews, we can assess and refine how the description structure and wording support (or not) learners with visual impairments. Interviews with learners indicate that these approaches are effective at providing information in a readily navigable format to learners with visual impairments, supporting learning with PhET sims [6-8].

Technical Infrastructure. We have also created the technical infrastructure necessary to allow PhET sims to communicate with assistive technologies. The HTML5 infrastructure used to create highly interactive sims, like PhET sims, does not come with built-in accessibility or any capabilities or standards for adding on accessibility features. Without significant effort, HTML5 sims cannot communicate with assistive devices and are inaccessible to students using assistive technology.

To address this technical challenge, we add an accessibility layer to the PhET sims that contains the accessibility content (e.g., alternative input structure, text descriptions, and sounds) and creates an interface between the visual sim and assistive technology. Referred to as the Parallel Document Object Model (PDOM), this layer consists of a hierarchical text-based representation of the sim. As a student uses the sim, the PDOM automatically updates to always reflect the sim's current state. The content 
in the PDOM appears as a typical HTML webpage to assistive technology allowing accessibility features to be implemented through international standards developed for webpages (e.g., [10]), and for learners to navigate the sim with common interactions they are already familiar with. In the John Travoltage sim, students can use common keyboard presses or screen reader shortcuts to navigate.

Collaboration. The PhET project has also begun organizing opportunities for community collaboration to share ideas, resources, and support for the work of creating inclusive interactive simulations. We started a11yinteractives ('a11y' is a common abbreviation for 'accessibility'), a community focused on sharing specific design and implementation challenges in monthly meetings, and sharing notable findings and news through an a11yinteractives listserve. We have also joined broader efforts for accessible learning resources, including leading an interactives-focused working group, organized by the DIAGRAM Center [9]. We also engage in frequent discussions with the accessibility technology and special education communities, where there is significant interest in utilizing accessible PhET sims as a customizable opensource resource, potentially relieving the burden of having to design from scratch an accessible prototype of a content-rich sims as research tools.

\section{CHALLENGES}

While we are proud of the advancements the PhET project, and others, have made to realize the vision of inclusive interactive resources for physics students with disabilities, the field is still in its infancy. Here we acknowledge some of the primary challenges (and resulting opportunities) that need to be addressed to allow for continued growth of the field.

Research. To address the needs of physics students with disabilities and their teachers, we need a better understanding of typical differentiation practices in inclusive classrooms, and corresponding analysis of what is effective and where gaps in resources exist. There are limited rigorous research findings on effective practices for supporting students with disabilities in learning physics concepts - in particular, students with sensory or mobility impairments. For example, how can sims like John Travoltage be incorporated into inclusive classrooms and coupled with physical demonstrations of static electricity in ways that are engaging and effective for students of all abilities? How might the accessibility features available in the sim be expanded or refined to support classroom use, how could the physical experiments be adaptable for different student needs, and what resources need to be provided to the teacher for effective planning and facilitation?
Additionally, foundational knowledge of assistive technology use is desperately needed, and difficult to collect. For our work with sims, we would like to know at what age most students with significant visual impairments have basic skills with keyboards and screen reader software. National trends in how students with visual impairments learn to use assistive technology is unknown. Whatever the trends are, they have likely changed drastically with the rise of tablet devices, making anecdotal accounts of regional training for those with vision impairment less useful.

There is a lack of research in this area due to a comparatively small amount of research inclusive of students with disabilities in STEM, few collaborative efforts at the intersection of special education and physics education, the logistical challenges in finding access to specific student populations and their teachers, and a lack of awareness of the rigorous research methodologies appropriate for studying small student populations (e.g, single-case study designs).

By sharing PhET's progress and opportunities for individuals in the physics education research community to engage with this area of need, we hope to encourage others to investigate opportunities for rigorous research in support of students with disabilities.

Design. Inclusive design of digital resources is a growing field. Using a one-size-fits-one approach, where resources are designed to be flexible and adapt to address unique needs of individuals, requires designing for overlapping layers (e.g., visuals, text description, sound), rather than for a single (typically visual) interface. In John Travoltage, the alternative input layer can support access for sighted students with mobility impairments, while also being a subcomponent of non-visual access (screen reader software is operated with the keyboard). When designing for alternative input, the use by sighted students and students with visual impairments need to be considered. When done effectively, the result is an intuitive experience for all learners. While anyone can learn inclusive design approaches, specialized expertise in the area is highly valuable, and difficult to find.

Additionally, as common computing devices evolve and expand modalities available (e.g., increasing ubiquity of speech interfaces, gesture, and haptic feedback), new opportunities for pedagogically powerful digital learning resources will emerge - for students of all abilities. Correspondingly, the design for seamless adaptation across input and output modalities will become increasingly sophisticated. For those interested in educational technologies, now is a great time to begin exploring the current capabilities for multi-modal digital resources in the classroom, and to experience the layered design techniques of inclusive design.

Technical Infrastructure. Multiple communities are working to address the technical issues related to inclusive 
interactives, but struggle with: 1) Working in silos or independently, without frequent and up-to-date knowledge about the needs of and progress by others. For example, web standards, browsers, and assistive technology developers are often unaware of education developers' and publishers' projects and needs. 2) Inequity in considering the needs of students with disabilities, as accessibility is often considered last in the development of educational resources, and is typically not weighted evenly with other factors, such as security or usability. 3) Lack of infrastructure to scale progress is often made in isolated pockets without a shareable infrastructure, limiting uptake and continued improvement by others.

The current amount of human and financial resources required to make a resource accessible, including developing foundational research to support well-designed and evidence-based resources, coupled with the high likelihood of limited distribution due to lack of awareness or knowledge of how to implement such resources prohibits the development of inclusive resources and significantly impedes inclusion of students with disabilities in physics classrooms. With coordinated efforts, we can change this dynamic and bring accessible, inclusive, practices into common usage by researchers, content creators, software developers, and ultimately teachers.

\section{POTENTIAL}

While creating accessible interactive sims will significantly impact students with disabilities, it also provides opportunities for innovations that support all learners and impact domains beyond physics education. Accessibility has a long history of leading to significant advancements in education, science, and healthcare [11]. For example, inventor Alexander Graham Bell was a teacher of deaf and deafblind students whose interest in sound stemmed from an effort to make sound visible to his students.
Additionally, there are corresponding new funding opportunities appearing, through the Dept. of Education's Office for Special Education Projects (OSEP) and Institute for Educational Sciences (IES), and the National Science Foundation's recent INCLUDES program solicitation which focuses on collective impact approaches to addressing issues of broadening participation - which includes broadening participation of students with disabilities.

If we work together to take advantage of this overlap in technological capability, legislative requirement, and funding opportunities, we can create a future where the capabilities of digital learning resources to provide effective, flexible, and personalized learning environments are fully realized. The result will be unprecedented access to highquality physics learning opportunities for all students, including students with disabilities, and truly inclusive classrooms.

\section{CONCLUSIONS}

The PhET project has made significant advancements in the development of inclusive interactive simulations. Through foundational research, inclusive design practices, development of new technical infrastructure, and facilitation of community collaboration, the PhET project is committed to the development of inclusive physics learning resources.

There is much work to be done, and we invite the physics education research community to consider where their research, creation of learning resources, and software development may overlap with the communities described here in working to support students with disabilities.

\section{ACKNOWLEDGEMENTS}

The authors thank the PhET team, our collaborators, and participants. This work is supported by the National Science Foundation DRL-1503439 and DRL-1621363, the Hewlett Foundation, and the University of Colorado Boulder.
[1] http://nationsreportcard.gov/science_2011/g8 nat.aspx? subtab_id=Tab_6\&tab_id=tab2\#chart. Retrieved 8/06/2014.

[2] Kahn, S. \& Lewis, A.R. (2014). JSTE, 25(8), 885-910.

[3] Kolowich, S. (2010). Inside Higher Ed. Retrieved from https://www.insidehighered.com/news/2010/06/30/kin dle. Retrieved 7/05/2017.

[4] http://phet.colorado.edu/. Retrieved 7/05/2017.

[5] http://phet.colorado.edu/en/simulation/travoltage. Retrieved 7/05/2017.

[6] Smith, T.L., Lewis, C. \& Moore, E.B. J Technol Pers Disabil. 5, 225-238.
[7] Moore, E.B., Smith, T.L. \& Randall, E., in Universal Access in Human-Computer Interaction. Users and Context Diversity. UAHCI 2016. Lecture Notes in Computer Science, edited by M. Antona and C. Stephanidis (Toronto, Canada, 2016), p. 112.

[8] Smith, T.L., Lewis, C. \& Moore, E.B., in Proceedings of the 18th International ACM SIGACCESS Conf on Computers and Accessibility (Reno, NV, 2016), p. 319.

[9] http://diagramcenter.org/accessible-dynamic-scientificgraphics.html. Retrieved 7/05/2017.

[10] https://www.w3.org/TR/wai-aria/. Retrieved 2/12/2016

[11] http://www.icdri.org/technology/ecceff.htm. Retrieved 5/18/2014 